\title{
Intencionalidad del embarazo y cuidados prenatales en Uruguay
}

\author{
Nicolás Brunet*, Wanda Cabella†, Alejandra Marroigł, Mathías Nathan§, \\ Ignacio Pardo", Guillermo Zoppolo**
}

\section{Resumen}

Introducción: desde hace algunos años el número de embarazos no intencionales en Uruguay ronda el $40 \%$; esta cifra es alta en comparación con otros países que también tienen baja fecundidad y da cuenta de las dificultades de acceso y uso eficaz de métodos anticonceptivos modernos. Además, varios estudios evidencian que los embarazos no intencionales están vinculados con cuidados prenatales insuficientes y peores desempeños de los recién nacidos respecto a los nacimientos resultantes de embarazos intencionales.

Objetivo: analizar la asociación entre intencionalidad de los embarazos y los cuidados prenatales en Uruguay a partir del estudio de su incidencia sobre la captación temprana del embarazo y las prácticas no saludables durante la gestación (fumar y tomar alcohol).

Método: se consideran los nacimientos no intencionales y a destiempo (no buscados en ese momento) como dos grupos de tratamiento y se comparan con el grupo de nacimientos intencionales. Se examina el efecto neto de la intencionalidad del embarazo sobre las prácticas de salud durante la gestación utilizando técnicas de Propensity Score Matching (PSM). Se utilizan datos provenientes de la Encuesta de Nutrición, Desarrollo Infantil y Salud (ENDIS), un estudio de panel que recoge información desde 2013 de madres con hijos de entre 0 y 3 años en hogares ubicados en localidades urbanas de Uruguay (mayores a 5.000 habitantes).

Resultados: antes de realizar el emparejamiento por PSM, las diferencias entre grupos de intención de embarazos fueron significativas para captación temprana y haber fumado, mientras que haber bebido alcohol no se asoció a diferencias significativas entre grupos de intención de los embarazos.

Palabras clave: Embarazo no deseado Atención prenatal Emparejamiento por puntaje de propensión

Key words: Pregnancy, unwanted

Prenatal care

Propensity score matching

\footnotetext{
* Profesor Adjunto. Instituto de Psicología de la Salud. Facultad de Psicología. Universidad de la República. † Profesora Agregada. Programa de Población, Facultad de Ciencias Sociales, Universidad de la República.

‡ Profesora Adjunta. Instituto de Economía-Instituto de Estadística. Facultad de Ciencias Económicas y de Administración. Universidad de la República. $\S$ Asistente de investigación. Programa de Población, Facultad de Ciencias Sociales, Universidad de la República.

TI Profesor Adjunto. Programa de Población, Facultad de Ciencias Sociales, Universidad de la República.

** Profesor Adjunto. Instituto de Estadística, Facultad de Ciencias Económicas y de Administración. Universidad de la República.

Correspondencia: Wanda Cabella. Correo electrónico: wanda.cabella@cienciassociales.edu.uy

Fuentes de financiamiento: este trabajo se inscribe en programa de investigación Entornos familiares, desempeños de los niños y políticas sociales, financiado por la Comisión Sectorial de Investigación Científica de la Universidad de la República a través del programa Grupos I+D 2014

Los autores declaran no tener conflicto de intereses.

Recibido: 8/6/20

Aprobado: 5/10/20
} 


\section{Introducción}

\section{Motivación y objetivo}

En los últimos años, los embarazos no intencionales que culminan en nacimientos se ubican en torno al $40 \%$ en Uruguay $^{(1)}$. Esta cifra, alta para un país con baja fecundidad, es consistente entre fuentes de datos distintas, como encuestas poblacionales y registros médicos.

El objetivo de este trabajo es profundizar en el análisis de la intencionalidad de los embarazos en Uruguay, con énfasis en el estudio de los vínculos entre intencionalidad del embarazo y cuidados prenatales (captación temprana del embarazo y prácticas saludables durante la gestación). Se consideran dos subcategorías de embarazos no intencionales: los embarazos no intencionales propiamente dichos (no buscados ni esperados en absoluto) y los embarazos a destiempo (no buscados en ese momento).

La asociación entre intencionalidad de los embarazos, desempeño de la gestación y resultados en la primera infancia ha sido sugerida por variedad de estudios ${ }^{(2-5)}$. Sin embargo, las dificultades derivadas de la calidad de los datos y de la capacidad de aislar el impacto de la intencionalidad respecto de otros factores entorpecen la observación de los efectos netos de esta relación. La estrategia de análisis aplicada en el presente estudio tiene como principal antecedente la utilizada por Kost y Lindberg $^{(6)}$. En ese trabajo se examina el efecto de la planificación del embarazo sobre los comportamientos de salud de la madre utilizando técnicas de Propensity Score Matching (PSM). El presente artículo adopta esta técnica de ponderación, incluyendo variables que puedan estar asociadas simultáneamente a la intencionalidad del embarazo y a los cuidados prenatales.

\section{Planificación e intencionalidad de los nacimientos}

La idea de fecundidad asociada a embarazos no deseados (unwanted fertility) fue popularizada en la década de $1960^{(7,8)}$. Su principal objetivo fue lograr estimaciones de la proporción de nacimientos no buscados, asumiendo que podrían haberse evitado en caso de contar con métodos anticonceptivos eficientes o acceso al aborto. Pero a pesar de su larga historia, no hay consenso sobre cuál es su conceptualización y medición más apropiada. La variedad de términos utilizados es elocuente a este respecto: se habla de embarazo no deseado, no buscado, no esperado, no planificado, no intencional. A pesar de que son evidentes los matices entre el deseo, búsqueda, planificación o intención de tener un hijo, solo en los últimos años se ha intentado consensuar la forma de registrar los embarazos que no surgen de una intención explícita de concebir.
Aun así, la intencionalidad de los embarazos sigue siendo un concepto difícil de construir y de medir ${ }^{(9-11)}$. Su indagatoria suele basarse en preguntas cerradas a propósito de intenciones, pero también de ideales o preferencias reproductivas, que pueden medirse prospectiva o retrospectivamente ${ }^{(12)}$. En este trabajo profundizamos en las preguntas de intenciones retrospectivas, aquellas que indagan sobre la fecundidad ya realizada, siguiendo el estándar utilizado en las Encuestas Demográficas y de Salud (DHS) adoptado en el cuestionario de la primera ronda de la Encuesta de Nutrición, Desarrollo Infantil y Salud (ENDIS 2013):

\section{¿Cuándo quedó embarazada...? \\ a. ...quería quedar embarazada en ese momento \\ b. ...más adelante \\ c. ...no quería tener (más) hijos}

Uno de los sesgos de esta pregunta es el efecto de racionalización ex post. Es decir, el sesgo introducido por la eventual revisión de la oportunidad del embarazo luego que ocurrió el nacimiento. Esta pregunta suele subestimar los nacimientos no planeados, en especial la respuesta "no quería estar embarazada" $"(11,12)$. Por ese motivo, se recomienda una medición multidimensional que trascienda el uso de una variable dicotómica (quería/no quería) e incluya factores como la intensidad del deseo de un hijo en una escala ordinal y la categoría de embarazos "antes de tiempo"(10,13), aunque no siempre está disponible la posibilidad de realizar estas mediciones.

La confiabilidad de la medición de la intencionalidad, cuando hay preguntas de encuesta limitadas, está dada por la consistencia de los resultados obtenidos en diferentes preguntas e incluso diferentes fuentes, como ocurre en el caso uruguayo.

\section{Los embarazos no intencionales en Uruguay}

El interés en el tema aumentó en las últimas décadas por múltiples motivos. La Conferencia Internacional de Población y Desarrollo de Naciones Unidas, celebrada en El Cairo en 1994, consagró la existencia de derechos reproductivos para todas las personas. Desde que la elección del número y el momento de tener hijos se consideran derechos, es aún más relevante conocer la magnitud de los embarazos no intencionales que terminan en nacimientos, puesto que, desde esta perspectiva, son indicativos de un ejercicio limitado de estos derechos. Simultáneamente, los programas de atención a la gestación y a la primera infancia despertaron un creciente interés en esta información, a medida que aumentaba la evidencia de vínculos entre la intencionalidad del embarazo y los cuidados prenatales y durante la primera 


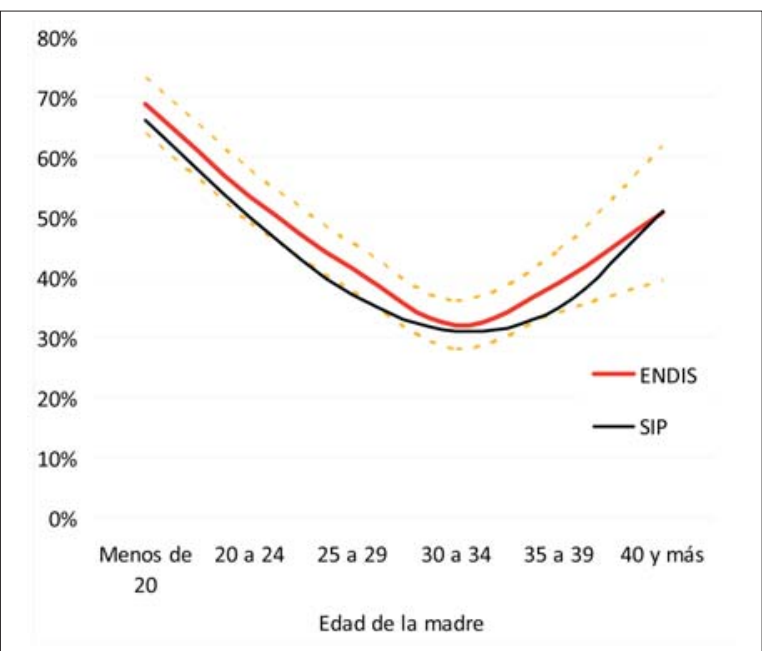

Figura 1. Embarazos no intencionales según el origen de información. Fuente: ENDIS 2013 y Sistema Informático Perinatal 2010-2013

infancia $^{(2,4)}$, e incluso en términos del impacto en el desarrollo de habilidades cognitivas y no cognitivas durante la infancia ${ }^{(14,15)}$.

En Uruguay, esas discusiones tienen una creciente importancia estratégica, considerando la proporción de mujeres que llevan a término embarazos no intencionales. Una proporción muy importante para un país con una fecundidad baja; la tasa global de fecundidad* cayó de 1,9 a 1,6 entre 2015 y 2018, pero el porcentaje de nacimientos no intencionales apenas descendió de $45 \%$ a $39 \%$.

De hecho, ENDIS 2013 encontró que el 47,7\% de las mujeres con hijos menores de 4 años no querían quedar embarazadas, porcentaje casi idéntico al obtenido en los datos del Sistema Informático Perinatal (SIP) para el año 2012 (46\%). Asimismo, se pudo observar que a menor edad de la madre al momento del parto y menor volumen de ingresos en el hogar aumentaba la probabilidad de haber cursado un embarazo no intencional ${ }^{(17)}$. La distribución por edad es asimismo casi idéntica en ambas fuentes (figura 1).

\section{Datos y métodos}

\section{Datos}

La información proviene de la ENDIS, una encuesta de panel implementada por el Instituto Nacional de Estadística. La primera ola, realizada en 2013, recogió in-

\footnotetext{
* La tasa global de fecundidad (TGF) se define como el "número de hijos que en promedio tendría una mujer de una cohorte hipotética de mujeres, que durante el período fértil no se encuentran expuestas a la mortalidad y están expuestas a las tasas de fecundidad de la población de estudio en el momento del cálculo"(16).
}

formación de madres de 3.077 niños y niñas de entre 0 y 3 años en hogares ubicados en localidades urbanas mayores a 5.000 habitantes de Uruguay. Nuestro universo de estudio incluye los nacimientos simples de mujeres de entre 20 y 44 años. Así, se analiza un total de 2.317* nacimientos.

El cuestionario de la ENDIS incluyó una pregunta para captar de manera retrospectiva si al momento de quedar embarazada**, la mujer: i) quería quedar embarazada en ese momento, ii) quería quedar embarazada más adelante, o iii) no quería tener (más) hijos. A partir de esta pregunta se clasificaron los nacimientos de las mujeres en función de tres grupos: nacimiento intencional, nacimiento a destiempo y nacimiento no intencional.

Para examinar el efecto de las intenciones del embarazo sobre los comportamientos de las madres, consideramos como variables de resultados*** si la mujer a) asistió a la primera consulta ginecológica durante el primer trimestre del embarazo (captación temprana); b) fumó durante el embarazo; c) tomó alcohol durante el embarazo $^{* * * *}$. En la tabla 1 se resumen las variables utilizadas en el análisis.

\section{Método}

Para lograr nuestro objetivo se utiliza la metodología de Emparejamiento por Puntaje de Propensión, conocida como Propensity Score Matching (PSM), que permite aislar el efecto de la intencionalidad del embarazo sobre los comportamientos maternos asociables a cuidados prenatales, siguiendo el procedimiento de Stuart ${ }^{(18)}$ y Ho y colaboradores ${ }^{(19)}$, basado en los trabajos pioneros de Rosebaum y Rubin ${ }^{(20)}$. Dado que las características sociodemográficas de las madres intervienen en esta relación, aplicar esta metodología permite aislar el efecto

* Los 2.317 casos representan el $75 \%$ de los 3.077 niños que entrevistó la ENDIS en la primera ola de relevamiento. La cantidad de casos analizada es menor por los siguientes motivos: encuestas no contestadas por la madre (110 casos, 3,6\%), embarazos múltiples, adoptados y otros (185 casos, 6\%) y madres menores de 20 o mayores de 44 años (465 casos, 15\%).

** La ENDIS solo recoge información correspondiente a embarazos que resultaron en un nacimiento.

*** Las variables de resultados consideradas son binarias; para seleccionarlas se adoptó un criterio similar al de Kost \& Lindberg ${ }^{(2)}$, basado en guías de salud del U.S. Department of Health and Human Services.

${ }^{* * * *}$ En Cabella et al ${ }^{(17)}$ se evidenció que según la ENDIS el número de mujeres que declaró haber asistido a la primera consulta durante el primer trimestre de embarazo estaba por encima de lo esperable de acuerdo a los registros del CNV y el Sistema Informático Perinatal (SIP), cuya información es más confiable por tratarse de registros realizados durante el embarazo y no de forma retrospectiva. Sin embargo, no se evidenció la existencia de sesgos en la distribución de las intenciones del embarazo y de las características sociodemográficas consideradas, según la sobredeclaración de haber hecho control durante el primer trimestre del embarazo. Además, realizamos el análisis de la captación temprana utilizando la variable del CNV: Ios resultados sustanciales no modifican la interpretación de los resultados. 
Tabla 1. Variables consideradas en el análisis.

\begin{tabular}{|c|c|c|}
\hline Variable & Categorías & Descripción \\
\hline \multicolumn{3}{|l|}{ Variable de tratamiento } \\
\hline \multirow[t]{5}{*}{ Intención } & & $\begin{array}{l}\text { Categoría de intención al momento de quedar embarazada del } \\
\text { niño/a (retrospectiva). }\end{array}$ \\
\hline & Nacimiento a destiempo & \\
\hline & (Quería, pero más adelante) & \\
\hline & Nacimiento intencional & \\
\hline & (No queria tener (más) hijos) & \\
\hline \multicolumn{3}{|c|}{ Covariables de emparejamiento } \\
\hline \multirow[t]{4}{*}{ Edad } & $20-24$ & Edad de la madre al momento del nacimiento \\
\hline & $25-29$ & \\
\hline & $30-34$ & \\
\hline & $35-44$ & \\
\hline \multirow[t]{3}{*}{ Orden de nacimiento } & 1 & Orden de nacimiento del hijo/a \\
\hline & 2 & \\
\hline & 3 y más & \\
\hline \multirow[t]{3}{*}{ Educación } & & $\begin{array}{l}\text { Años de educación aprobados por la madre al momento de la } \\
\text { encuesta }\end{array}$ \\
\hline & De 10 a 12 & \\
\hline & 13 y más & \\
\hline \multirow[t]{2}{*}{ Situación de pareja } & & $\begin{array}{l}\text { Aproximación a la situación conyugal de la madre al nacimiento } \\
\text { del hijola. Los casos en los que el padre biológico nunca vivió }\end{array}$ \\
\hline & Sin pareja / Situación de pareja frágil & $\begin{array}{l}\text { con el niño/a o dejó de vivir con él antes de que cumpliera } 6 \\
\text { meses, son clasificados como "situación de pareja frágil" }\end{array}$ \\
\hline \multirow[t]{2}{*}{ Institución } & Privada & Tipo de establecimiento de atención del parto \\
\hline & Pública & \\
\hline \multirow[t]{2}{*}{ Región } & Montevideo & Región de residencia habitual de la madre \\
\hline & Resto del pais & \\
\hline \multicolumn{3}{|l|}{ Variables de resultado } \\
\hline No captación temprana & No & $\begin{array}{l}\text { Realización del primer control de embarazo durante el primer } \\
\text { trimestre ( } 1 \text { si no se realiza el control en el primer trimestre, } 0 \\
\text { en caso contrario) }\end{array}$ \\
\hline \multirow[t]{2}{*}{ Fumar } & No & $\begin{array}{l}\text { Fumó durante el embarazo ( } 1 \text { si declaró que fumó durante el } \\
\text { embarazo, } 0 \text { en caso contrario) }\end{array}$ \\
\hline & SI & \\
\hline \multirow[t]{2}{*}{ Tomar alcohol } & No & $\begin{array}{l}\text { Bebió bebidas alcohólicas durante el embarazo ( } 1 \text { si declaró } \\
\text { que bebió alcohol durante el embarazo, } 0 \text { en caso contrario)* }\end{array}$ \\
\hline & Sí & \\
\hline \multicolumn{3}{|c|}{$\begin{array}{l}\text { Fuente: ENDIS } 2013 \text { y Certificado del Nacido Vivo. } \\
\text { * Cabe recordar que las variables de resultado relativas al consumo de tabaco y alcohol durante el embarazo se obtienen por autorrepor- } \\
\text { te, lo que puede subrepresentar la proporción de mujeres que llevan adelante esas conductas. Sin embargo, un estudio realizado en Uru- } \\
\text { guay con datos del SIP no detectó un sesgo de declaración respecto al consumo de tabaco entre las embarazadas que concurrían a con- } \\
\text { trolarse }{ }^{(19)} \text {. }\end{array}$} \\
\hline
\end{tabular}




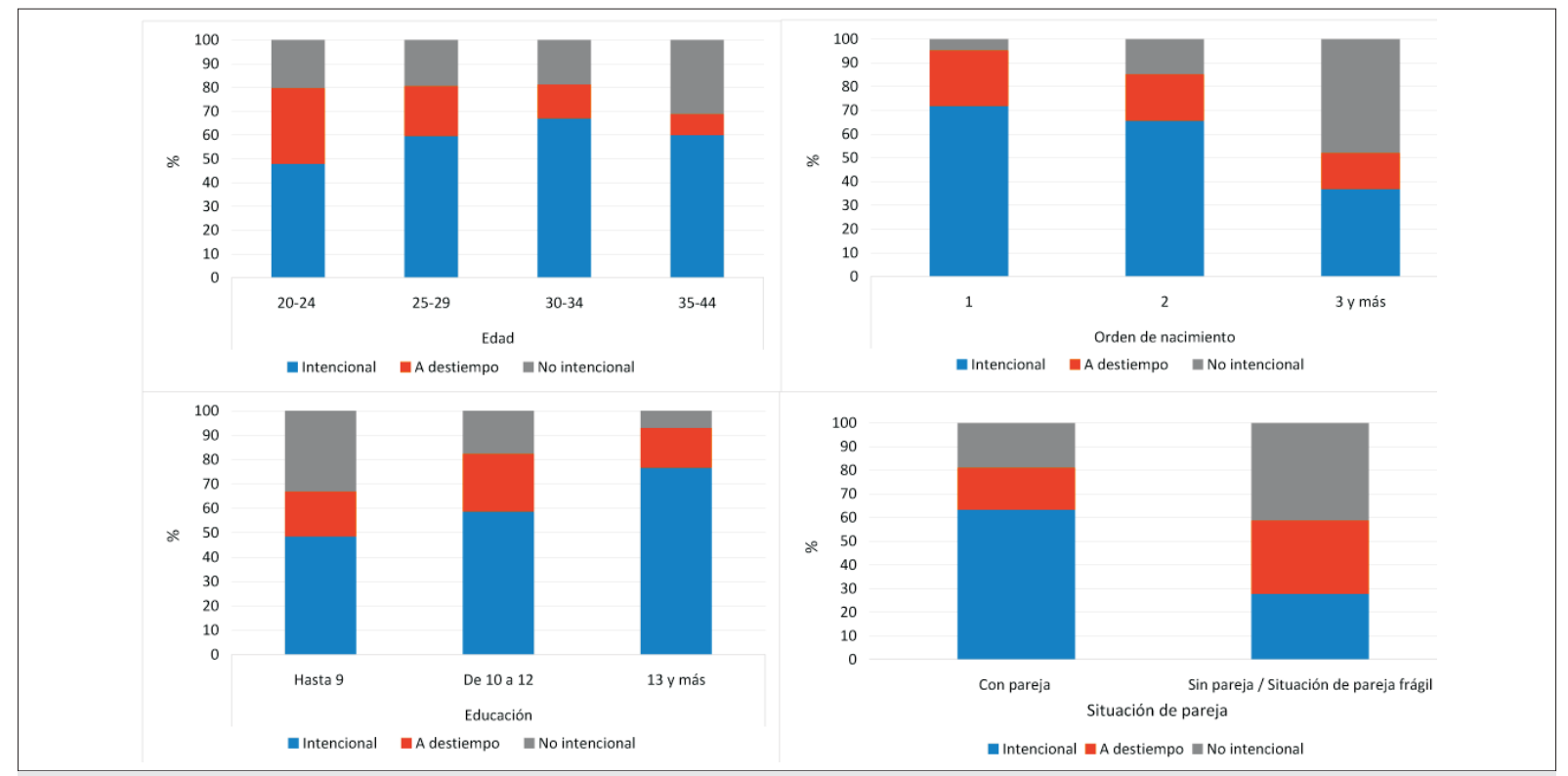

Figura 2. Intencionalidad de los nacimientos según atributos del nacimiento y la madre. Fuente: ENDIS 2013.

de las intenciones sobre los comportamientos maternos, anulando la influencia de características sociodemográficas como la edad materna, el orden de nacimiento y el nivel educativo materno, entre otras.

En primer lugar, se definen las covariables para realizar el emparejamiento entre los nacimientos de los distintos grupos de intención (tabla 1), considerando aquellas que la bibliografía documenta como relacionadas con las variables de resultados. El método de PSM asume que no existen diferencias entre grupos en otras características (por ejemplo, inobservables). Esto se conoce como supuesto de "ignorabilidad" (ignorability) en la asignación al tratamiento ${ }^{(20)}$. No es posible contrastar empíricamente el cumplimiento de este supuesto, ya que se requiere independencia condicional de las características inobservables con la asignación a los grupos. La literatura recomienda incorporar en el emparejamiento todas las variables relacionadas con la pertenencia a dichos grupos y con las variables de resultado. Sin embargo, la inclusión de variables redundantes puede generar problemas en la varianza de las estimaciones ${ }^{(18)}$.

En segundo lugar, se utiliza la distancia exacta entre las covariables como medida de semejanza entre casos de distintos grupos. Esto implica que los casos de grupos diferentes se emparejan si tienen iguales valores en todas las covariables consideradas. Este procedimiento es ideal cuando el número de casos perdidos y la cantidad de covariables utilizadas para emparejar no son muy elevados (ver tabla a.1.1 en Anexo). Además, resulta recomendable cuando las variables para el emparejamiento son binarias ${ }^{(18,22)}$. En tercer lugar, se utiliza el método exacto en donde se emparejan casos de diferentes gru- pos cuando todas las covariables tienen igual valor ${ }^{(22)}$. Cada subclase emparejada* puede contener distinto número de casos de cada grupo de intenciones, lo que hace a este método flexible y óptimo al minimizar el promedio de las distancias entre los grupos dentro de las subclases emparejadas ${ }^{(18,19)}$.

Para el análisis de resultados y estimación del efecto de las intenciones, dado el emparejamiento anterior, se consideraron los nacimientos no intencionales y a destiempo como dos grupos de tratamiento y se compararon con el grupo de nacimientos intencionales. Primero de forma independiente, luego con comparaciones entre los grupos de tratamiento ${ }^{(23)}$. También se consideraron los nacimientos no intencionales y a destiempo juntos y se los comparó con el conjunto de nacimientos intencionales.

Los resultados en este trabajo corresponden a las estimaciones del efecto promedio del tratamiento conocido como average treatment effect (ATE), que corresponde a la diferencia de medias de las variables de resultado entre los grupos emparejados. Este efecto se estima a partir de las diferencias de medias entre los grupos de intención emparejados, es decir, ponderados y condicionales a iguales características entre grupos y su significación estadística se contrasta mediante un t de Student pareado. El análisis de los datos y las estimaciones se realizaron utilizando el software libre $\mathrm{R}$.

* En nuestro caso hay un total de 288 subclases, determinadas por los valores de las covariables consideradas. 


\section{Resultados}

\section{El perfil sociodemográfico de la intencionalidad de los embarazos}

En primer lugar se muestra la distribución de la intencionalidad en función del perfil sociodemográfico, que toma en consideración la evidencia acumulada sobre el tema respecto a que la intencionalidad de los nacimientos está fuertemente relacionada con los atributos de la madre y del contexto del nacimiento en general (figura 2).

Nuestros resultados muestran la relación existente entre la edad de la madre y la probabilidad de cursar un embarazo no intencional que culmine en nacimiento. La evidencia acumulada hace esperable que la no intencionalidad se distribuya en forma de $\mathrm{U}$, como la que se observa en la figura 1: más alta al principio y al final de la vida reproductiva que en las edades centrales. Algo así sucede con la muestra de la ENDIS, aun cuando la edad mínima está en 20 años y no en la adolescencia, lo que hubiera hecho más pronunciada la no intencionalidad temprana. Aquí, a diferencia de la figura 1, pueden verse diferenciadamente nacimientos no intencionales propiamente dichos y a destiempo. El pico de intencionalidad sucede en las madres de 30 a 34 años y las más jóvenes son en mayor medida quienes consideran el nacimiento como ocurrido a destiempo, lo que resulta razonable en términos de expectativas asociadas al curso de vida.

Otro hallazgo esperable es la correlación entre intencionalidad y lugar en la estratificación social, reforzando hallazgos previos ${ }^{(24,25)}$. En las figuras puede verse a partir de las variables de nivel educativo, pero también se lo podría ver si la variable elegida fuese el tipo de institución en que cursaron el parto (ver resultados en tabla a.2 del Anexo). En ambos casos, los atributos asimilables a posiciones menos ventajosas (baja educación, institución pública) se asocian a una mayor proporción de nacimientos no intencionales o a destiempo.

El contexto del nacimiento también diferencia la probabilidad de que el nacimiento sea o no intencional. En primer lugar, el orden de nacimiento: mientras que los primeros nacimientos son intencionales en más de $70 \%$, apenas un poco más de un tercio de los nacimientos de orden 3 o más lo son. Pero más importante que esto es el contexto conyugal, que hace el doble de probable la intencionalidad de un embarazo si la madre está en pareja que si no la tiene, o si tiene una situación de pareja frágil. Por el contrario, no hay diferencias significativas entre Montevideo y el resto del país, reafirmando que el territorio no es un clivaje especialmente significativo para los diferentes comportamientos demográficos en Uruguay (ver resultados en tabla a.2 del Anexo).

\section{Efectos de las intenciones de los embarazos sobre comportamientos maternos}

Finalmente, para aislar el efecto de las intenciones sobre las variables de resultado, se emparejaron los casos de los distintos grupos utilizando las covariables y el método explicado anteriormente. Primero, cabe observar la distribución univariada de las variables sociodemográficas de la madre y de los nacimientos, utilizadas para emparejar los grupos, dentro de cada categoría de intencionalidad (tabla 2).

Los resultados muestran que distribuciones de las covariables de emparejamiento condicionales a los grupos de intención son diferentes y, por tanto, resultan variables relevantes para el emparejamiento. Como fue descrito en la sección metodológica, con el emparejamiento exacto se pueden calcular las diferencias de medias ponderadas entre los grupos y condicionales a iguales características (covariables de emparejamiento).

Una vez generado el emparejamiento, es posible estimar los efectos de los dos grupos de nacimientos no intencionales por separado (no intencionales propiamente dichos y a destiempo) en comparación con los intencionales. También comparar nacimientos no intencionales y a destiempo y, por último, realizar la comparación clásica de nacimientos no intencionales y a destiempo en relación con los intencionales.

Así, en la tabla 3 se muestran los efectos de la intencionalidad sobre los comportamientos maternos durante el embarazo: ausencia de captación temprana, fumar y tomar alcohol. Antes de realizar el emparejamiento por PSM, las diferencias entre grupos de intención de embarazos resultaban significativas para captación temprana y haber fumado, mientras que haber bebido alcohol no se asociaba a diferencias significativas entre grupos de intención de los embarazos. Al realizar la comparación de las variables de resultado sin emparejar por características observables de los grupos, $16 \%$ de los embarazos no intencionales no muestran captación temprana, mientras que este porcentaje asciende a $3 \%$ entre los embarazos intencionales (diferencia de 13 puntos porcentuales, $\mathrm{p}<0,05)$. Además, para los embarazos a destiempo la no realización de controles en el primer trimestre corresponde a $9 \%$ de los embarazos de este grupo y presenta una diferencia significativa (diferencia de 6 puntos porcentuales, $\mathrm{p}<0,05$ ) con el $3 \%$ correspondiente al grupo de embarazos intencionales sin emparejamiento por características observables. Por su parte, el porcentaje de mujeres que fuman durante el embarazo en el grupo de embarazo no intencional es de $27 \%$, en tanto que este porcentaje es de $12 \%$ en el grupo de embarazos intencionales (diferencia significativa, $\mathrm{p}<0,05$ ). Además, el porcentaje que fumó dentro del grupo de embarazos a des- 
Tabla 2. Distribución de variables sociodemográficas de la madre y el nacimiento condicional a los grupos de intención.

\begin{tabular}{|c|c|c|c|c|c|c|}
\hline & & \multicolumn{5}{|c|}{ Grupos de intención } \\
\hline & & 1 & $A D$ & $\mathrm{NI}$ & Total & $N$ \\
\hline \multicolumn{2}{|l|}{ Intención del nacimiento } & 59 & 19 & 22 & 100 & 2.317 \\
\hline \multirow[t]{4}{*}{ Edad } & $20-24$ & 20 & $41^{*}$ & $23^{\dagger}$ & 25 & 573 \\
\hline & $25-29$ & 28 & 30 & 25 & 28 & 644 \\
\hline & $30-34$ & 31 & $20^{*}$ & 23 & 27 & 628 \\
\hline & $35-44$ & 21 & $9^{*}$ & $29^{*, \dagger}$ & 20 & 472 \\
\hline \multirow[t]{3}{*}{ Orden de nacimiento } & 1 & 40 & 39 & $7^{*, \dagger}$ & 32 & 748 \\
\hline & 2 & 41 & 36 & $24^{*, \dagger}$ & 36 & 834 \\
\hline & 3 y más & 20 & $24^{*}$ & $69^{*, \dagger}$ & 31 & 722 \\
\hline \multirow[t]{3}{*}{ Educación } & Hasta 9 & 37 & $43^{*}$ & $68^{*, \dagger}$ & 45 & 1.044 \\
\hline & De 10 a 12 & 29 & $35^{*}$ & $23^{*, \dagger}$ & 29 & 665 \\
\hline & 13 y más & 34 & $22^{*}$ & $8^{*, \dagger}$ & 26 & 605 \\
\hline \multirow[t]{2}{*}{ Situación de pareja frágil } & Con pareja & 94 & $79^{*}$ & $76^{*}$ & 87 & 2.018 \\
\hline & Sin pareja & 6 & $21^{*}$ & $24^{*}$ & 13 & 299 \\
\hline \multirow[t]{2}{*}{ Institución } & Privada & 72 & $58^{*}$ & $44^{*, \dagger}$ & 64 & 1.458 \\
\hline & Pública & 28 & $42^{*}$ & $56^{*, \dagger}$ & 36 & 838 \\
\hline \multirow[t]{2}{*}{ Región } & Montevideo & 61 & 59 & 65 & 61 & 1.421 \\
\hline & Resto del país & 39 & 41 & 35 & 39 & 896 \\
\hline \multicolumn{7}{|c|}{$\begin{array}{l}\text { Nota: porcentajes suman } 100 \text { al interior de los grupos de intención. } \\
\text { I: Intencional; AD: A destiempo; NI: No intencional. } \\
* \mathrm{p}<0,05 \text { (paired t test vs. Intencional). } \\
\dagger \mathrm{p}<0,05 \text { (paired t test vs. A destiempo). } \\
\text { I: Intencionales; AD: A destiempo; NI: No intencionales. } \\
\text { Fuente: ENDIS 2013. }\end{array}$} \\
\hline
\end{tabular}

tiempo es de $17 \%$, que también resulta significativamente distinto $(\mathrm{p}<0,05)$ al grupo de embarazos intencionales.

Tras la aplicación del PSM, las diferencias se mantienen significativas para la captación temprana, tanto en la comparación entre intencionales y no intencionales, como entre intencionales y a destiempo, mientras que los nacimientos no intencionales y a destiempo no muestran diferencias significativas entre sí.

Así, puede decirse que las madres que tuvieron embarazos no intencionales o a destiempo presentaron captación temprana en menor proporción que las que vivieron embarazos intencionales. Los resultados muestran que en los embarazos no intencionales hay 8 puntos porcentuales más de embarazos que no se captan tempranamente respecto a los intencionales $(\mathrm{p}<0,05)$ una vez emparejados los grupos, es decir, descontando los efectos de las características observables consideradas. Para los embarazos a destiempo esta proporción es de 3 puntos porcentuales mayor $(\mathrm{p}<0,05)$ que los intencionales, aun cuando se haya controlado por las características observables de interés. Dado que estas diferencias existen aun cuando se controla por las covariables, podemos asumir que esta mayor probabilidad de captación temprana se explica por factores vinculados a la intencionalidad de los embarazos. Algo similar puede decirse (aunque restringido a la diferencia entre los embarazos intencionales y los no intencionales, sin involucrar los que fueron a destiempo) en cuanto al hábito de fumar durante el embarazo. 
Tabla 3. Resultados en cuidados prenatales según intencionalidad del nacimiento. Grupos emparejados por PSM, Uruguay 2013.

\begin{tabular}{|c|c|c|c|c|}
\hline & $\mathrm{NI}$ & I & Diferencia & $N$ \\
\hline No captación temprana & 0,16 & 0,03 & $0,13^{*}$ & 1.831 \\
\hline Fumó & 0,27 & 0,12 & $0,15^{*}$ & 1.836 \\
\hline \multirow[t]{2}{*}{ Bebió alcohol } & 0,13 & 0,1 & 0,02 & 1.832 \\
\hline & $A D$ & 1 & & $N$ \\
\hline No captación temprana & 0,09 & 0,03 & $0,06^{*}$ & 1.782 \\
\hline Fumó & 0,17 & 0,12 & $0,05^{*}$ & 1.787 \\
\hline \multirow[t]{2}{*}{ Bebió alcohol } & 0,12 & 0,1 & 0,02 & 1.782 \\
\hline & $\mathrm{NI}$ & $A D$ & & $N$ \\
\hline No captación temprana & 0,16 & 0,09 & $0,07^{*}$ & 935 \\
\hline Fumó & 0,27 & 0,17 & $0,1^{*}$ & 937 \\
\hline \multirow[t]{2}{*}{ Bebió alcohol } & 0,13 & 0,12 & 0,01 & 932 \\
\hline & $A D \circ N I$ & 1 & & $\mathrm{~N}$ \\
\hline No captación temprana & 0,13 & 0,03 & $0,1^{*}$ & 2.274 \\
\hline Fumó & 0,22 & 0,12 & $0,1^{*}$ & 2.280 \\
\hline Bebió alcohol & 0,12 & 0,1 & 0 & 2.273 \\
\hline \multicolumn{5}{|l|}{ Resultados emparejados } \\
\hline & $\mathrm{NI}$ & 1 & & $N$ \\
\hline No captación temprana & 0,12 & 0,03 & $0,08^{*}$ & 1.415 \\
\hline Fumó & 0,22 & 0,14 & $0,08^{*}$ & 1.420 \\
\hline \multirow[t]{2}{*}{ Bebió alcohol } & 0,16 & 0,12 & 0,04 & 1.417 \\
\hline & $A D$ & 1 & & $N$ \\
\hline No captación temprana & 0,06 & 0,03 & $0,04^{*}$ & 1.584 \\
\hline Fumó & 0,13 & 0,13 & $-0,01$ & 1.589 \\
\hline \multirow[t]{2}{*}{ Bebió alcohol } & 0,09 & 0,11 & $-0,02$ & 1.584 \\
\hline & $\mathrm{NI}$ & $A D$ & & $N$ \\
\hline No captación temprana & 0,14 & 0,12 & 0,03 & 727 \\
\hline Fumó & 0,26 & 0,22 & 0,04 & 729 \\
\hline \multirow[t]{2}{*}{ Bebió alcohol } & 0,14 & 0,14 & 0 & 726 \\
\hline & $A D \circ N I$ & 1 & & $N$ \\
\hline No captación temprana & 0,1 & 0,03 & $0,06^{*}$ & 2.089 \\
\hline Fumó & 0,17 & 0,14 & $0,03^{*}$ & 2.095 \\
\hline Bebió alcohol & 0,11 & 0,12 & 0 & 2.089 \\
\hline
\end{tabular}




\section{Discusión}

Este trabajo aporta nueva evidencia de la influencia de la intencionalidad de los embarazos sobre comportamientos como la captación temprana y el hábito de fumar. De acuerdo con nuestros resultados esta relación existe y amerita estudiarse en mayor profundidad. La inclusión de una categoría intermedia de intencionalidad (embarazo a destiempo) en el análisis de los efectos de la intencionalidad sobre las prácticas durante la gestación evidencia que la introducción de sintonía fina en la medición de la intencionalidad puede redundar en una mayor capacidad de capturar la complejidad de esta relación. En concreto, se sugiere considerar: 1) la incorporación de mediciones adicionales, más cercanas al momento del parto (dado que las intenciones iniciales pueden transformarse ante la inminencia del nacimiento) y 2) la modificación o adición de preguntas para captar mejor lo ambiguo o difuso de las intenciones. Por ejemplo, diferenciando las personas que estaban buscando activamente un embarazo de aquellas que no. La incorporación al SIP del formato de tres categorías de la ENDIS constituiría en sí mismo un importante avance en el registro de la intencionalidad de los embarazos en las estadísticas de nacimientos.

También podría avanzarse en la incorporación de nuevas variables de resultados, es decir, en el conocimiento de otros comportamientos asociados a los cuidados prenatales, como el consumo de drogas ilícitas, el uso sistemático de suplementos vitamínicos indicados durante la gestación y en general las prácticas que favorecen o entorpecen el buen desempeño gestacional de acuerdo con las indicaciones médicas vigentes.

No siempre pueden verificarse estas relaciones hipotetizadas (como en el caso del consumo de alcohol, en este trabajo), lo que lleva a discutir en qué medida la ausencia de impacto observado obedece a la escasa capacidad del análisis de datos para aislar la intencionalidad, a la propia ausencia de efecto alguno, o la inadecuación de las fuentes de datos disponibles para detectar efectos relativamente pequeños.

De todos modos, lo más relevante, en los casos en que se comprueba una relación entre intencionalidad y cuidados prenatales, es dar un paso más en el estudio del vínculo causal e indagar en los mecanismos o canales a través de los cuales un atributo subjetivo, como el recogido en la intención reproductiva declarada, influye en los cuidados prenatales. Para eso, es necesario discutir qué está expresando la intencionalidad en términos de comportamiento de las madres y en qué dimensiones de su vida (emocional/afectiva, material, conyugal) podría estar impactando, para que a su vez afecten los comportamientos durante el embarazo.
Estas inquietudes son parte de la agenda de investigación sobre el tema, que se beneficiaría también con la inclusión de un espectro más amplio de atributos personales (rasgos de personalidad, modelos de relaciones conyugales o restricciones específicas en las condiciones de vida), dado que allí se podrían observar configuraciones que generen al mismo tiempo embarazos no intencionales y la propensión a ciertos comportamientos prenatales.

En la especificación de modelos, como el presentado en este trabajo, se incorporan variables de control con la intención de aislar el efecto de la intencionalidad y especificar correctamente este tipo de relaciones, pero dimensiones no observables de los datos podrían reflejar una heterogeneidad relevante para la explicación.

Finalmente, el aporte de los estudios cualitativos podría ofrecer otros indicios de interés, aportando datos de contexto que describan los significados de la maternidad, edades normativas a las que tener hijos o expectativas de cuidados infantiles luego del nacimiento. Ello permitiría complementar los datos brindados por los indicadores y eventualmente auxiliar la generación de indicadores más refinados y modelos causales más exhaustivos para precisar la explicación del fenómeno.

\section{Summary}

Introduction: in Uruguay, the number of unintended pregnancies has been around $40 \%$ for several years. This is rather a high percentage if compared to other countries who also have low fertility rates and evidences difficulties in access to modern contraceptive methods or using them effectively. Likewise, several studies evidence unintended pregnancies are related to insufficient antenatal care services and worse outcome in the new-borns when compared to births resulting from intentional pregnancies.

Objective: to study the relationship between pregnancy intentionality and antenatal care services in Uruguay, based on an analysis of its impact on the early engagement of pregnancies and non-healthy practices during pregnancy (smoking and alcohol consumption).

Method: unintended and untimely births (not sought at that time) were included in the study as two treatment groups, and they are compared to the group of intentional births.

The net effect of pregnancy intention on health practices during pregnancy was examined using the de Propensity Score Matching (PSM) techniques. We used data delivered by the Nutrition, Child Development and Health Survey, a panel study that has been collecting information from mothers of children between 0 and 3 years old who live in urban localities of Uruguay (with over 5,000 inhabitants) since 2013. 
Results: Prior to the PSM matching, the differences in early engagement and smoking were significant between pregnancy intention groups, whereas alcohol consumption was not associated to significant differences between pregnancy intention groups.

\section{Resumo}

Introdução: há anos, o número de gestações não intencionais no Uruguai gira em torno de $40 \%$; este número é alto em comparação com outros países que também têm baixa fecundidade e explica as dificuldades de acesso e uso de métodos anticoncepcionais modernos. Além disso, diversos estudos mostram que a gravidez não intencional está associada ao pré-natal insuficiente e piores parâmetros do recém-nascido em comparação com o nascimento decorrente da gravidez intencional.

Objetivos: analisar a associação entre intencionalidade da gravidez e assistência pré-natal no Uruguai, a partir do estudo de sua incidência na detecção precoce da gravidez e práticas não saudáveis durante a gravidez (tabagismo e etilismo).

Método: os nascimentos não intencionais e prematuros (não desejados no momento) são considerados dois grupos de tratamento e comparados com o grupo de partos intencionais. O efeito líquido da intencionalidade da gravidez sobre as práticas de saúde durante a gravidez é examinado usando técnicas de correspondência de pontuação de propensão (PSM). São utilizados os dados da Pesquisa de Nutrição, Desenvolvimento Infantil e Saúde (ENDIS), um estudo de painel que coleta informações desde 2013 de mães com filhos de 0 a 3 anos em domicílios localizados em áreas urbanas do Uruguai (mais de 5.000 habitantes).

Resultados: antes de realizar a comparação por PSM, as diferenças entre os grupos de intenção de gravidez foram significativas para recrutamento precoce e tabagismo, enquanto ter bebido álcool não foi associado a diferenças significativas entre os grupos de intenção de gravidez.

\section{Bibliografía}

1. Cabella W, Nathan M, Pardo I. La caída de la fecundidad en Uruguay entre 2015 y 2018. En: Fondo de Población de las Naciones Unidas; Ministerio de Salud Pública; Universidad de la República. Descenso acelerado de la fecundidad en Uruguay entre 2015 y 2018. Tres estudios para su análisis. Montevideo: UNFPA, MSP, UdelaR, 2019:33-69.

2. Kost K, Lindberg L. Pregnancy intentions, maternal behaviors, and infant health: investigating relationships with new measures and propensity score analysis. Demography 2015; 52(1):83-111.
3. Dibaba Y, Fantahun M, Hindin M. The effects of pregnancy intention on the use of antenatal care services: systematic review and meta-analysis. Reprod Health 2013; 10:50.

4. Gipson JD, Koenig MA, Hindin MJ. The effects of unintended pregnancy on infant, child, and parental health: a review of the literature. Stud Fam Plann 2008; 39(1):18-38.

5. Mohllajee AP, Curtis KM, Morrow B, Marchbanks PA. Pregnancy intention and its relationship to birth and maternal outcomes. Obstet Gynecol 2007; 109(3):678-86.

6. Kost K, Landry DJ, Darroch JE. Predicting maternal behaviors during pregnancy: does intention status matter? Fam Plann Perspect 1998; 30(2):79-88.

7. Harkavy O, Jaffe FS, Wishik SM. Family planning and public policy: who is misleading whom? Science 1969; 165(3891):367-73.

8. Blake J. Population policy for Americans: is the government being misled? Science 1969; 164:522-9.

9. Koenig MA, Acharya R, Singh S, Roy TK. Do current measurement approaches underestimate levels of unwanted childbearing? Evidence from rural India. Popul Studies (Camb) 2006; 60(3):243-56.

10. Santelli JS, Lindberg LD, Orr MG, Finer LB, Speizer I. Toward a multidimensional measure of pregnancy intentions: evidence from the United States. Stud Fam Plann 2009; 40(2):87-100.

11. Bongaarts J. The measurement of wanted fertility. Popul Develop Rev 1990; 16(3):487-506.

12. Casterline JB, El-Zeini LO. The estimation of unwanted fertility. Demography 2007; 44(4):729-45.

13. Pearson E, Biswas K, Chowdhury R, Sultana S, ShahiduIlah M, Moreau C. Multidimensional measures of fertility intentions regarding terminated pregnancy and association with subsequent reproductive health outcomes in Bangladesh. En: Population Association of America. Annual Meeting, San Diego-EEUU, 2015.

14. de la Rochebrochard E, Joshi H. Children born after unplanned pregnancies and cognitive development at 3 years: social differentials in the United Kingdom millennium cohort. Am J Epidemiol 2013; 178(6):910-20.

15. Joyce TJ, Kaestner R, Korenman S. The effect of pregnancy intention on child development. Demography 2000; 37(1):83-94.

16. Uruguay. Instituto Nacional de Estadística. Definiciones indicadores demográficos. Disponible en: http://www.ine. gub. uy/indicadores-demograficos1 [Consulta: 16 febrero 2020].

17. Cabella W, De Rosa M, Failache P, Fitermann P, Katzkowicz N, Medina M, et al. Salud, nutrición y desarrollo en la primera infancia en Uruguay. Primeros resultados de la ENDIS. Montevideo: UCC, Mides, 2015.

18. Stuart EA. Matching methods for causal inference: a review and a look forward. Stat Sci 2010; 25(1):1-21.

19. Ho D, Imai K, King G, Stuart E. MatchIt: nonparametric preprocessing for parametric causal inference. J Stat Softw 2011; 42(8):1-28. 
20. Rosenbaum PR, Rubin DB. The central role of the propensity score in observational studies for causal effects. Biometrika 1983; 70:41-55.

21. Harris $\mathbf{J}$, Balsa A, Triunfo $\mathbf{P}$. Tobacco control campaign in Uruguay: impact on smoking cessation during pregnancy and birth weight. J Health Econ 2015; 42:186-96.

22. Hansen BB. Full matching in an observational study of coaching for the SAT. J Am Stat Assoc 2004; 99(467):609-18.

23. Imbens GW. The role of the propensity score in estimating dose-response functions. Biometrika 2000; 87(3):706-10.
24. Amarante V, Cabella W. La brecha entre la fecundidad deseada y la observada en Montevideo y su Área Metropolitana. Notas poblac 2015; (100):11-34

25. Peri A, Pardo I. Nueva evidencia sobre la hipótesis de la doble insatisfacción en Uruguay: ¿cuán lejos estamos de que toda la fecundidad sea deseada? (Serie Divulgación). Montevideo: UNFPA, 2008.

\section{Contribución de autores}

Ignacio Pardo, https://orcid.org/0000-0002-6185-1082. Concepción. Diseño. Redacción y revisión crítica.

Nicolás Brunet, https://orcid.org/0000-0003-1881-0258. Concepción. Diseño.

Wanda Cabella, https://orcid.org/0000-0002-8522-7866. Concepción. Diseño. Redacción y revisión crítica.

Alejandra Marroig, https://orcid.org/0000-0003-0716-174X. Diseño, ejecución, análisis. Interpretación de resultados. Redacción y revisión crítica.

Mathías Nathan, https://orcid.org/0000-0002-3549-283X. Concepción. Diseño.

Guillermo Zoppolo, https://orcid.org/0000-0001-8979-3163. Análisis. Interpretación de resultados. 


\section{Anexos - Calidad del emparejamiento}

La calidad del emparejamiento para el método de emparejamiento exacto se evalúa a través de la cantidad de casos emparejados, a partir de los cuales se realiza el análisis de diferencias en las variables de resultado relevantes. En la siguiente tabla se observa que el emparejamiento dentro de cada grupo de intención recupera siempre al menos un $73 \%$ de los casos no emparejados. El mínimo de casos emparejados aparece únicamente cuando se realizan dos comparaciones: No intencionales vs Intencionales para el grupo Intencionales, y en No intencionales vs A destiempo para el grupo A destiempo. Para el resto de las comparaciones se empareja al menos un $77 \%$ de los casos.

Tabla A.1.1 Pérdida de casos por no emparejamiento - Exact Matching

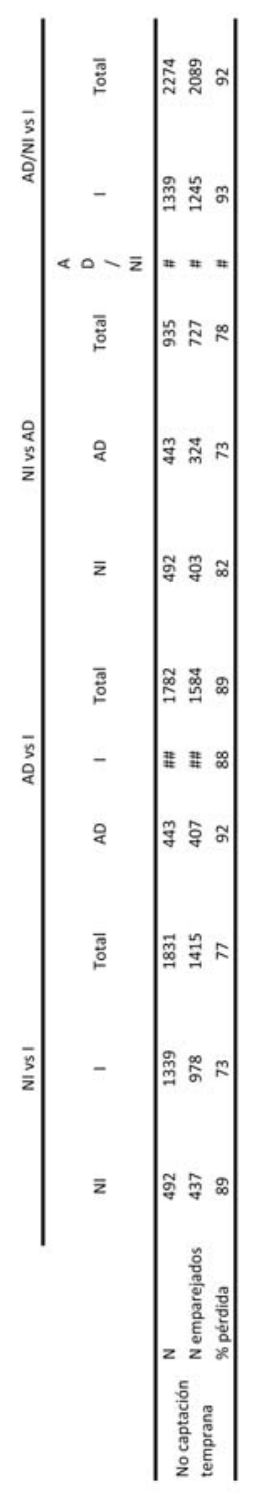

Nota: I: Intencionales; AD: A destiempo; NI: No intencionales.

$\mathrm{Al}$ analizar las otras variables de resultado, haber fumado (Fumar) y haber bebido alcohol (Alcohol) durante el embarazo, los casos analizados cambian levemente por datos faltantes en estas variables.

Fuente: ENDIS 2013. 
Para las cuatro comparaciones de grupos realizadas en este trabajo se presentan las características sociodemográficas de las madres y de los nacimientos de aquellos que no fueron emparejados por el método exacto de emparejamiento. En la siguiente tabla se pueden apreciar las diferencias existentes entre los grupos en los casos no emparejados.

Tabla A.1.2 Distribución condicional y no condicional de las características de casos no emparejados.

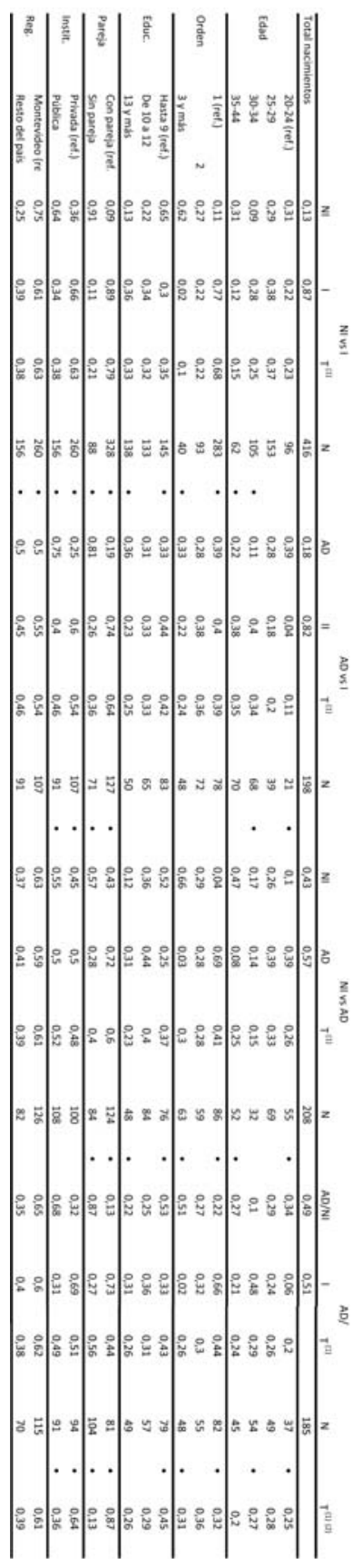

Nota: Los porcentajes suman 100 dentro de los grupos de intenciones (columnas).

(1) Distribución no condicional a los grupos de las características.

(2) Esta columna presenta la distribución no condicional para todas las madres según tabla $1(\mathrm{~N}=2.317)$

- $\mathrm{p}<0,05$ (paired ttest)

Fuente: ENDIS 2013. 
Tabla a.2. Intencionalidad de los nacimientos según atributos del nacimiento y la madre.

\begin{tabular}{|c|c|c|c|c|c|}
\hline & & $\begin{array}{c}\text { Intencional } \\
(\%)\end{array}$ & $\begin{array}{c}\text { A destiempo } \\
(\%)\end{array}$ & $\begin{array}{c}\text { No intencional } \\
(\%)\end{array}$ & N \\
\hline \multirow{4}{*}{ Edad } & $20-24$ & 47,8 & 31,9 & 20,2 & 573 \\
\hline & $25-29$ & 59,6 & 21,0 & 19,4 & 644 \\
\hline & $30-34$ & 67,0 & 14,3 & 18,6 & 628 \\
\hline & $35-44$ & 60,0 & 8,9 & 31,1 & 472 \\
\hline \multirow{3}{*}{$\begin{array}{l}\text { Orden de } \\
\text { nacimiento }\end{array}$} & 1 & 71,8 & 23,5 & 4,7 & 748 \\
\hline & 2 & 65,7 & 19,5 & 14,8 & 834 \\
\hline & 3 y más & 36,8 & 15,2 & 47,9 & 722 \\
\hline \multirow{3}{*}{ Educación } & Hasta 9 & 48,5 & 18,5 & 33,1 & 1044 \\
\hline & De 10 a 12 & 58,8 & 23,6 & 17,6 & 665 \\
\hline & 13 y más & 76,7 & 16,4 & 6,9 & 605 \\
\hline \multirow{3}{*}{$\begin{array}{l}\text { Situación de } \\
\text { pareja }\end{array}$} & Con pareja & 63,4 & 17,7 & 18,9 & 2018 \\
\hline & Sin pareja / & & & & \\
\hline & $\begin{array}{l}\text { Situación de } \\
\text { pareja frágil }\end{array}$ & 27,8 & 31,1 & 41,1 & 299 \\
\hline \multirow{2}{*}{ Institución } & Pública & 44,5 & 22,4 & 33,1 & 1458 \\
\hline & Privada & 67,4 & 17,7 & 15,0 & 838 \\
\hline \multirow{2}{*}{ Región } & Montevideo & 59,5 & 20,5 & 20,0 & 1421 \\
\hline & Resto del país & 58,3 & 18,7 & 22,9 & 896 \\
\hline
\end{tabular}

Fuente: ENDIS 2013. 